\title{
Pulmonary Tuberculosis Accompanying Adenocarcinoma of Lung in a Young Female from India
}

\author{
Nandita Pal ${ }^{1}$ \\ ${ }^{1}$ Assistant Professor, Dept. of Microbiology, College of Medicine and Sagore Dutta Hospital, Kamarhati, \\ Kolkata, 700058, India
}

\begin{abstract}
Present communication describes a case of a young female patient suffering from pulmonary tuberculosis associated with delayed diagnosis of adenocarcinoma of lung. Timely diagnosis requires a high index of suspicion and knowledge of the spectrum of clinical and radiologic features. This case report emphasizes the importance of diagnostic evaluation like thoracic computed tomography scan (CT) and CT guided fine needle aspiration cytology (FNAC) which if used without much delay, may lead to accurate diagnosis of the fatal disease without undue delay.
\end{abstract}

Keywords- adenocarcinoma of lung, CT guided fine needle aspiration cytology, pulmonary tuberculosis

\section{Introduction}

Adenocarcinoma is the most common lung cancer in nonsmokers and in women. ${ }^{[1]}$ Timely diagnosis requires a high index of suspicion and knowledge of the spectrum of clinical and radiologic features. This is also the most frequent type of tumor detected in low-dose CT screening trials. ${ }^{[2]}$ FNAC technique should be used early and more frequently to shorten the diagnostic interval and allow more prompt therapy for persistent lung lesions. ${ }^{[3]}$ Present communication mentions a case of young, seemingly healthy, female patient suffering from pulmonary tuberculosis accompanied with lung adenocarcinoma.

\section{Case History}

A twenty-three year old female patient attended hospital with a three month history of irregular fever and productive cough. In the past she had been treated now and then with various antibiotic drugs for respiratory tract infections. There was no contact or family history and no history of smoking or alcohol abuse. No lymphadenopathy was found on examination. In the lower portion of the left lung field dullness to percussion was noted. In addition, diminished breath sounds and diminished vocal resonance were appreciated. Otherwise the systemic examination was non-contributory. Chest X-ray showed apical left lung infiltrations with evidence of pleural effusion in lower zone of left side (Fig: 1). Sputum, examined on three consecutive days, revealed acid-fast bacilli (AFB). Patient was seronegative for Human immunodeficiency virus (HIV). Gram staining of sputum did not reveal any microorganism. Pleural fluid analysis was not done at this stage.

Following therapy with anti-tubercular drugs, for two months; thoracic CT scan was done which revealed nodular opacity of $9 \mathrm{~mm}$ diameter in lower zone of left lung (Fig: 2). Transcutaneous CT guided FNAC findings showed nests of atypical cells with enlarged nuclei, prominent nucleoli, and eosinophilic cytoplasm suggestive of poorly differentiated solid pattern adenocarcinoma. Immunohistochemistry finding was inconclusive. Patient was put on chemotherapeutic regimen, but did not respond and ultimately succumbed to her illness six weeks later.

\section{Discussion}

Pleural effusion is a well known consequence of pulmonary tuberculosis, but in this case; the fluid shadow probably concealed the nodular opacity of a malignant lesion in chest $\mathrm{x}$-ray. Prior visualization of the lung nodule could have prompted early investigations. Pleural fluid analysis was also not done earlier; or the adenocarcinoma may have been detected earlier. Since the patient was so young, so the possibility of carcinoma was remote and also the finding of AFB in sputum seemed a justified explanation for the presenting signs and symptoms, which caused the undue delay in getting the CT thorax and FNAC done. In our patient; CT thorax and FNAC confirmed the diagnosis of adenocarcinoma of lung.

Here, malignancy presented as a solitary nodular lesion in peripheral lung parenchyma. ${ }^{[4]}$ The patient did not present with any obvious symptom of lung adenocarcinoma as such because of the small size and peripheral location of the lesion. Approximately $40-50 \%$ of solitary pulmonary nodules are malignant most of which are adenocarcinoma. ${ }^{[5]}$ Past history of recurrent respiratory tract infections in this patient probably indicates the immunocompromised state of the patient owing to the accompanying malignancy. 
Adenocarcinoma is the most common lung cancer in nonsmokers, women and in young age group. ${ }^{[1]}$ In this case, it could not be assured weather the patient had primary lung adenocarcinoma as such or the nodule visualized in thoracic CT scan was a secondary metastasis from extra-thoracic source; because of inconclusive immunohistochemistry findings. The recurrence of respiratory tract infections and occurrence of pulmonary tuberculosis in a young, well-nourished, urban patient, from good socio-economic strata, without any contact or family history; should have aroused the suspicion of underlying serious disease much earlier.

\section{Conclusion}

High index of suspicion and early utilization of diagnostic measures is very much needed in such cases. Late detection of the disease in this case caused early mortality of the patient.

\section{Figures:}

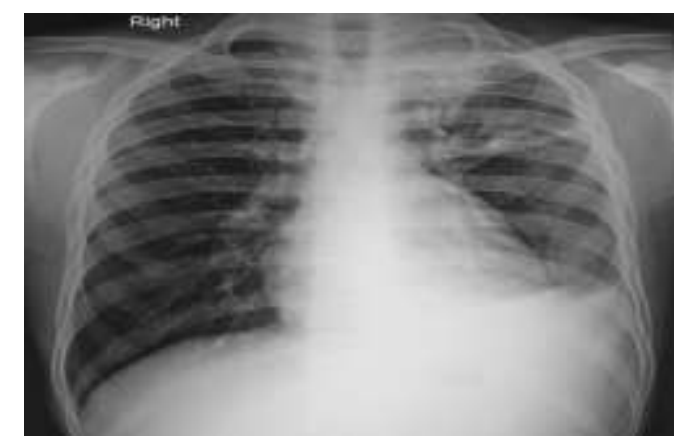

Fig 1: Chest X-ray showed apical left lung infiltrations with evidence of pleural effusion in lower zone of left side

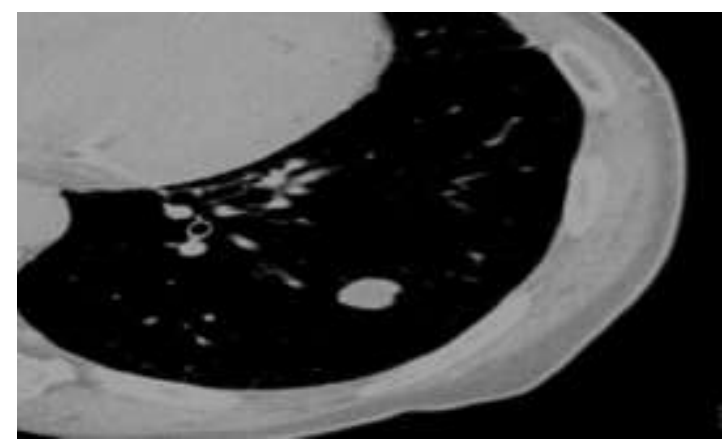

Fig 2: Chest CT scan revealed a 9 mm nodule in left lower lobe of the lung

\section{References:}

[1]. Subramanian J, Govindan R. Lung cancer in never smokers: a review. Journal of Clinical Oncology 2007;25 (5): 561-70.

[2]. Pelosi G, Sonzogni A, Veronesi G, et al. Pathologic and molecular features of screening low-dose computed tomography (LDCT)detected lung cancer: A baseline and 2-year repeat study. Lung Cancer. 2008;62:202-14.

[3]. Gangopadhyay M, Chakrabarti I, Ghosh N, Giri A. Computed tomography guided fine needle aspiration cytology of mass esions of lung: Our experience. Indian J Med Pediatr Oncol 2011;32:192-6 Lee HY, Han J, Lee KS, Koo JH, Jeong SY, Kim BT, Cho YS, Shim YM, Kim J, Kim K, Choi YS. Lung adenocarcinoma as a solitary pulmonary nodule: prognostic determinants of CT, PET, and histopathologic findings. Lung Cancer 2009;66(3):379-85.

[4]. Lee HY, Han J, Lee KS, Koo JH, Jeong SY, Kim BT, Cho YS, Shim YM, Kim J, Kim K, Choi YS. Lung adenocarcinoma as a solitary pulmonary nodule: prognostic determinants of CT, PET, and histopathologic findings. Lung Cancer 2009;66(3):379-85.

[5]. Gould MK, Fletcher J, Iannettoni MD, et al. Evaluation of patients with pulmonary nodules: when is it lung cancer?: ACCP evidence-based clinical practice guidelines (2nd ed). Chest 2007;132(3):108-30. 